\title{
Atmospheric Neutrino Flux Measurement
}

\author{
Kimihiro Okumura ${ }^{1, *}$ \\ ${ }^{1}$ Research Center for Cosmic Neutrino (RCCN), Institute for Cosmic Ray Research (ICRR), University of Tokyo, \\ 5-1-5 Kashiwa-no-ha, Kashiwa, Chiba, Japan
}

\begin{abstract}
The atmospheric neutrino flux has been measured by several experiments and its understanding has been improved by comparing with the theoretical predictions in detail. In this paper recent results of the flux measurements will be reviewed and discussed.
\end{abstract}

\section{Introduction}

Atmospheric neutrinos are the end particles produced by meson decay in air showers, initiated by cosmic ray interactions with the atmosphere. They are most abundant in nature above sub-GeV energies. The fluxes on the Earth are well calculated by several simulation studies using the inputs of the measured cosmic ray flux and the hadronic interactions. However, the neutrino flux predictions are affected by several factors, such as the primary cosmic ray flux, compositions, hadron interactions, atmosphere model, seasonal variations, geomagnetic effects, etc. The flux predictions can be tested directly by atmospheric neutrino flux measurements.

Generally atmospheric neutrinos are produced by the decay of particles such as pions $(\pi)$ and kaons $(K)$, and dominate below several hundred TeV. Those are called as the "conventional" atmospheric neutrino flux. The nominal energy spectrum follows a power-law with spectral index of $-3.7, d N / d E \propto E^{-3.7}$. The flavor ratio of muon and electron neutrinos, $\left(v_{\mu}+\bar{v}_{\mu}\right) /\left(v_{e}+\bar{v}_{e}\right)$, is about two around 1 $\mathrm{GeV}$ where pion decay neutrinos are dominant. Above $O(10) \mathrm{GeV}$ the $v_{e}$ flux becomes smaller by the steeper spectrum since the parent muons $(\mu)$ reach ground level without decay. Instead of the $\mu / \pi$-decay neutrino, $K$-decay neutrino becomes dominant because of its faster decay time constant. The flux prediction of $K$-decay neutrino largely differs depending on the $K$ production models, causing the major flux uncertainties in this energy range.

As the energy increases further above several ten $\mathrm{TeV}$, $K$ mesons also reaches to the ground without decay and the conventional neutrino flux is suppressed. Instead the "prompt" flux produced by $D$ meson particles begins to dominate. The understanding of the prompt neutrino is important because they could be a substantial background of the astrophysical neutrino observation. The flux predictions based on the simulation are available but its detection has not yet been successfully done.

The primary cosmic rays such as protons and helium are deflected by the Earth's geomagnetic field, and suffer

\footnotetext{
*e-mail: okumura@icrr.u-tokyo.ac.jp
}

from the rigidity cutoff depending on the detector site below several ten GeV. Therefore the so called "East-West" anisotropy, which is the flux modulation depending on the azimuthal direction, is observed in atmospheric neutrinos as well as in cosmic rays. The anisotropy is anticipated to be increased in the $\mathrm{GeV}$ region and near horizontal directions. The feature of the geomagnetic effect in the flux simulation, such as the bending of the primary and secondary cosmic ray particles, can be tested with the atmospheric neutrino data.

The cosmic ray flux is known to be anti-correlated with solar activity, which cycles in an 11 year period, since the cosmic rays entering the Earth are scattered out by the solar wind. Accordingly the modulation of the atmospheric neutrino flux bythe solar cycle is expected, especially in the low energies below $10 \mathrm{GeV}$ and in the direction of the polar regions.

The understanding of the the atmospheric neutrino flux is absolutely imperative because the atmospheric neutrino itself is utilized for the measurement of neutrino oscillations. Also atmospheric neutrinos could be a background of rare event search such as proton decay, dark matter, astronomical neutrinos, etc. The background level is estimated based on the flux prediction. The flux measurement can give a severe test directly to the flux predictions, and make it more reliable.

\section{Methodology}

Basically the atmospheric neutrino flux is estimated by counting neutrino events produced by charged-current (CC) interactions with detector mass. In order to measure the flavor dependent flux, the particle identification capability of the out-going lepton is required. Also neutralcurrent (NC) events could be a background, and the data quality of the flux sample depends on the rejection performance of $\mathrm{NC}$ backgrounds. In addition the measured flux is affected by neutrino oscillations below $100 \mathrm{GeV}$, and the Earth absorption at $O(10) \mathrm{TeV}$ and higher. Such effects should be taken into account. 

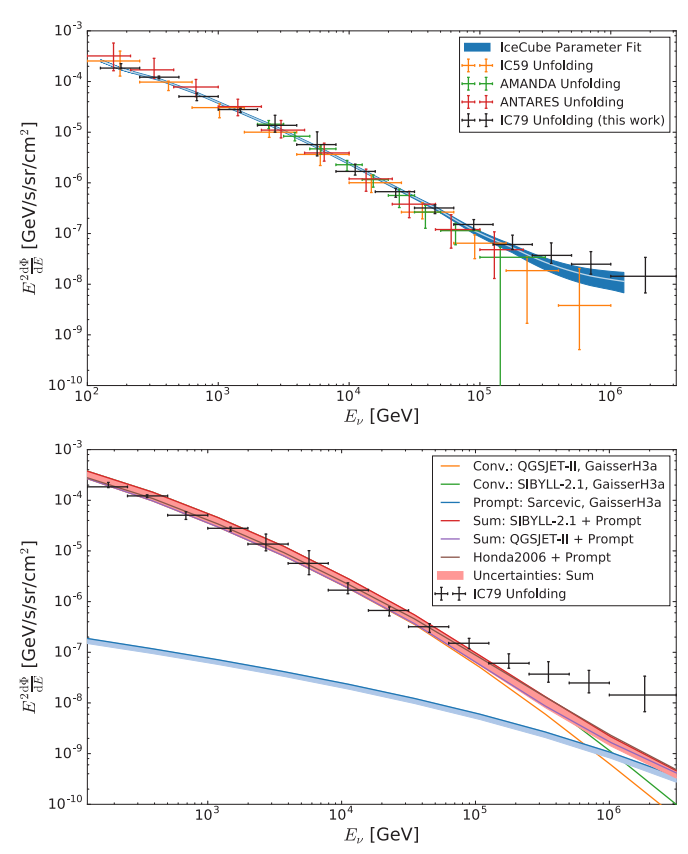

Figure 1. The atmospheric muon neutrino flux measured by several experiments from $100 \mathrm{GeV}$ to several $\mathrm{PeV}$ (upper), and the comparison of IceCube measurements and the theoretical predictions of conventional and prompt fluxes (lower). Figures are taken from [1].

In this paper the results of the flux measurement will be discussed in two categories, which are roughly grouped by the detector mass scale; the first category is kiloton scale detectors, such as Frejus and Super-Kamiokande (Super$\mathrm{K}$ ), measuring the flux below $\mathrm{TeV}$, mainly $\mathrm{GeV}$ energies. Super-K detector is equipped with the fine granularity of the photo-sensors for observing the detailed event topology providing excellent capabilities of particle identification and NC background rejection. Another category is kilometer cubic $\left(\mathrm{km}^{3}\right)$ scale detectors such as AMANDA, ANTARES, IceCube, using natural ice in the South Pole and the water of Mediterranean sea as the detector material. These detectors have sensitivities to measure smaller fluxes at energies greater than $O(100) \mathrm{GeV}$, thanks to the huge detector mass. However, due to the coarser arrangement of the photo-sensors, the event identification capability is not as good as compared to that of kiloton scale detectors. As a result, though the relatively good quality of data is provided for $v_{\mu}$ samples by identifying the muon track in the event, the contamination of NC background events is inevitable for $v_{e}$ measurement due to similar event topology.

\section{Flux measurement above $\mathrm{TeV}$ energies}

The flux of muon neutrino $\left(v_{\mu}\right)$ above $\mathrm{TeV}$ energies is well studied by several experiments, as shown in Figure 1. Basically upward-going $v_{\mu}$ sample are utilized for flux estimation to reduce the cosmic muon backgrounds. The measured spectra are covered from $O(100) \mathrm{GeV}$ up to several $\mathrm{PeV}$, and consistent with each other and also with theoretical predictions. Meanwhile the recent IceCube result

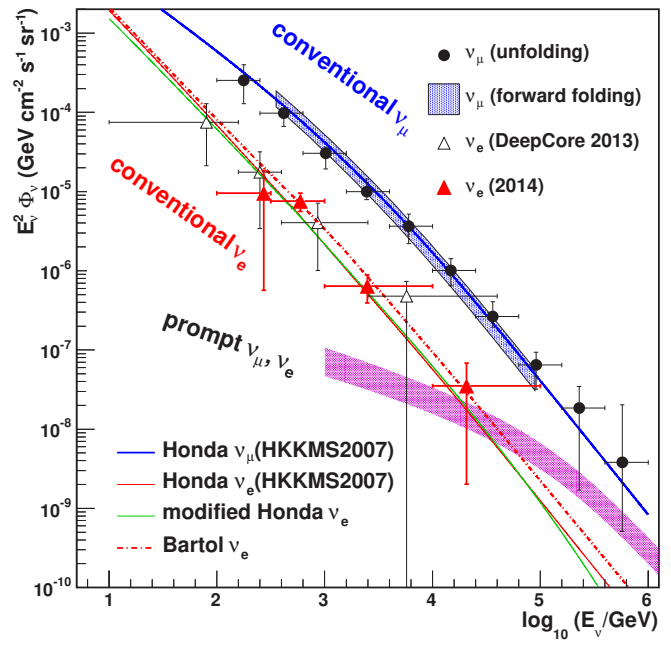

Figure 2. The atmospheric $v_{e}$ flux results by IceCube shown with the flux predictions. Figure is taken from [2].

shows a clear excess beyond $100 \mathrm{TeV}$ [1]. The excess is too large to be explained by the prompt component, and thought to be due to the external origin neutrinos produced by astronomical high energy objects in the universe.

Figure 2 shows the $v_{e}$ energy spectrum measured by the IceCube experiment. The measurement of $v_{e}$ flux is important because there are still large flux uncertainties in the flux calculation above $O(100) \mathrm{GeV}$ due to $K$ production cross section error in hadronic interactions. Since the contributions from $K$ decay is dominant rather than for $\pi$ decay neutrinos in this energy range, this result gives constraint on $K$ originated flux. Also the detection of the prompt flux component is expected by measuring the $v_{e}$ spectrum in the high energy end above $O(10) \mathrm{TeV}$ since the prompt flux begins to exceed the conventional one. Actually, $v_{e}$ measurement in $\mathrm{km}^{3}$ detectors is not easy due to NC backgrounds. A sophisticated event selection using the likelihood method is employed to discriminate $v_{e}$ events in the IceCube experiment [2]. The observed $v_{e}$ flux shows about $30 \%$ higher than flux prediction, which indicates a larger $K$ flux component than expected. No evidence of the prompt flux is obtained from the fitting result using the observed flux and spectrum shape.

\section{Flux measurement below $\mathrm{TeV}$ energies}

Figure 3 shows the measured $v_{\mu}$ and $v_{e}$ energy spectra by the Super-K experiment compared with flux predictions and other experimental results [3]. Super-K data covers the wider energy range from sub-GeV up to several $\mathrm{TeV}$, providing the significantly improved flux data. At the higher energy end the energy spectrum overlaps with $v_{\mu}$ measurements by $\mathrm{km}^{3}$ detectors, and the measured energy spectra are consistent and smoothly connected between lower and higher energy ranges. Figure 4 shows the detailed comparison of the measured fluxes with the flux predictions. They are consistent with each other within estimated uncertainties. The total systematic errors are estimated to 


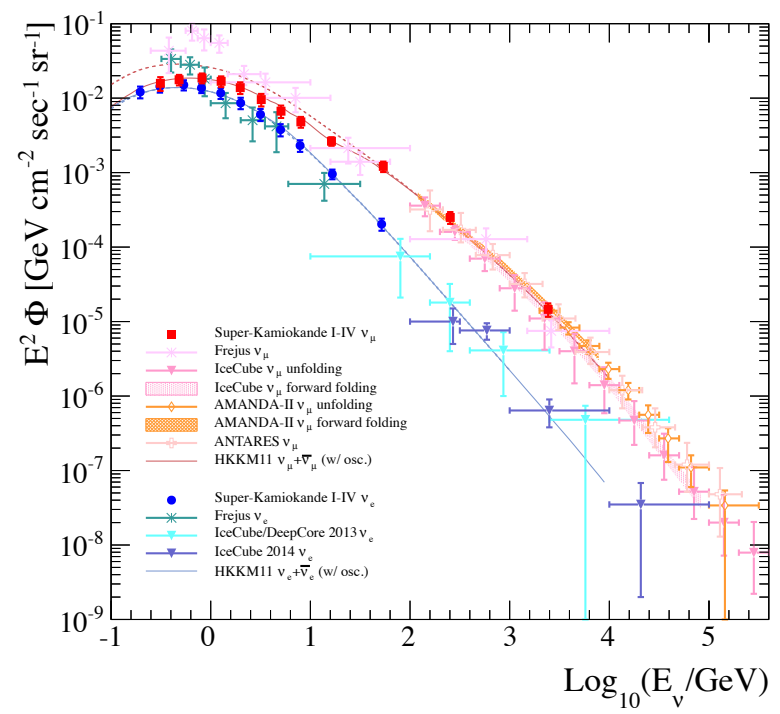

Figure 3. The measured energy spectra of the atmospheric $v_{e}$ and $v_{\mu}$ fluxes by Super-Kamiokande, shown with measurements by other experiments.

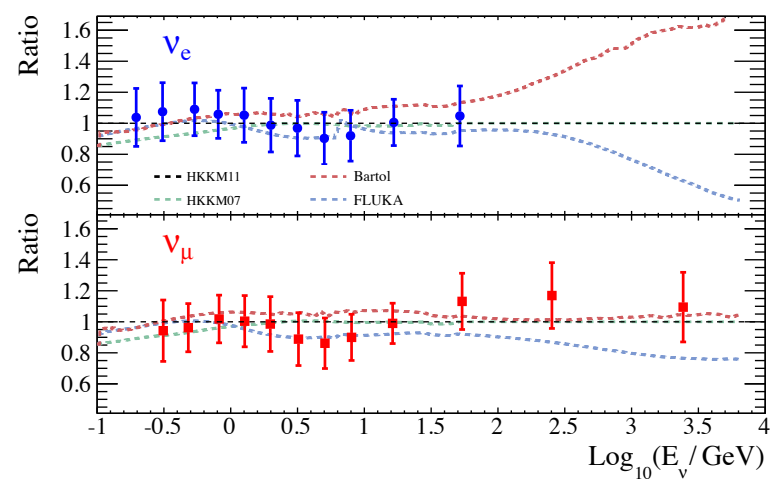

Figure 4. Measured energy spectra compared to the flux model predictions (dotted lines) from the HKKM11 (black), HKKM07 (light green), Bartol (red), and FLUKA (light blue) models. The vertical axis represents the flux ratio from the data (or each model) to HKKM11. Error bars include both statistical and systematic uncertainties.

be approximately $20 \%$, which comes dominantly from the uncertainties related to neutrino interactions.

Figure 5 shows the azimuthal spectra of Super-K $v_{e}$ (e-like) and $v_{\mu}$ ( $\mu$-like) sample. Those event samples are chosen to be optimal for getting the largest significance of the anisotropy. Clear modulations depending on the azimuthal direction are seen for both $e$-like and $\mu$-like data, which significances are $6.0 \sigma$ and $8.0 \sigma$, respectively. The azimuthal asymmetry are also investigated quantitatively to validate the implementation of the geomagnetic effect in the flux simulation. Two parameters, $A$ and $B$, are introduced for measuring the azimuthal modulation, representing the degree of the flux asymmetry between East and West directions, and the phase of the modulation curve, respectively. These parameter values are compared between data and expectation in several energies and zenith directions, as shown in Figures 6 and 7. The measured data
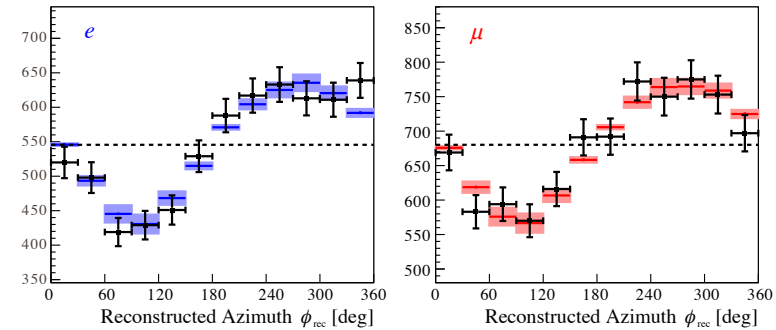

Figure 5. The azimuthal distributions of a subselection of $e$-like (left) and $\mu$-like (right) events, from the Super-K data (points with statistical error) and MC simulations (boxes with systematic error). The subselection is optimized to obtain the highest significance of the final A parameters, by using only events with $0.4<E_{\text {rec }}<3.0 \mathrm{GeV}$ and $\left|\cos \theta_{\text {rec }}\right|<0.6$.
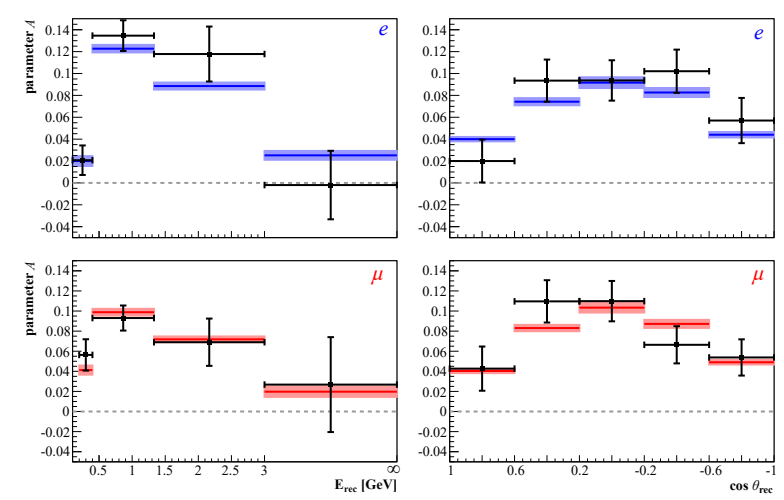

Figure 6. The parameter, $A$, depending on the reconstructed energy (left) and zenith angle (right), for $e$-like (upper) and $\mu$-like (lower) events, from the Super-K data (points with statistical error) and MC simulations (boxes with systematic error). A grey dotted line is drawn at $A=0$, the case of no east-west asymmetry, as a visual guide. The highest energy bin in the left plots is unbounded from above.
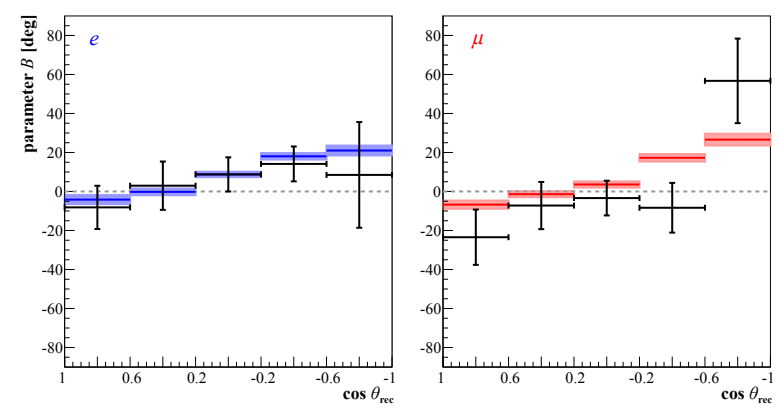

Figure 7. The parameter, $B$, depending on the cosine of the zenith angle, for $e$-like (left) and $\mu$-like (right) events, from the Super-K data (points with statistical error) and MC simulations (boxes with systematic error).

agree well with the predictions, confirming the implementation of the geomagnetic field in the flux calculation.

Super-K has performed a search for the correlation with solar activity using the atmospheric neutrino data in the period covering almost two solar cycles, as shown in Figure 8. The neutron monitor count, $C$, which is known 


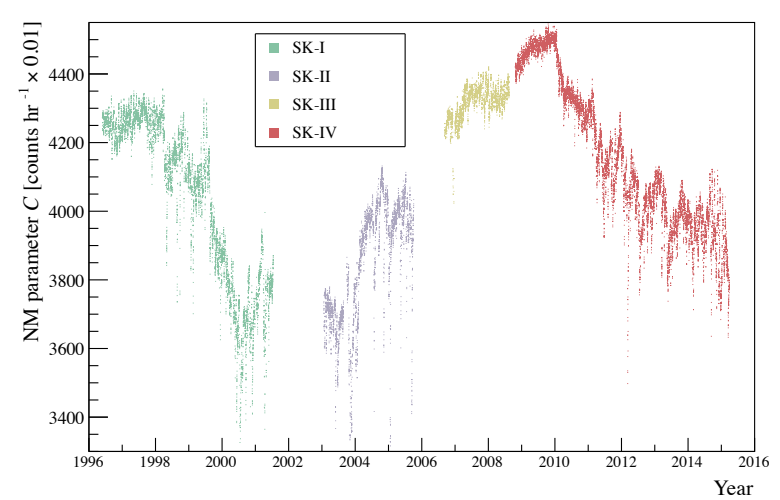

Figure 8. The neutron monitor parameter, $C$, over the Super$\mathrm{K}$ operational period, where low values of $C$ correspond to high solar activity, showing that almost two solar cycles are covered by the Super-K data
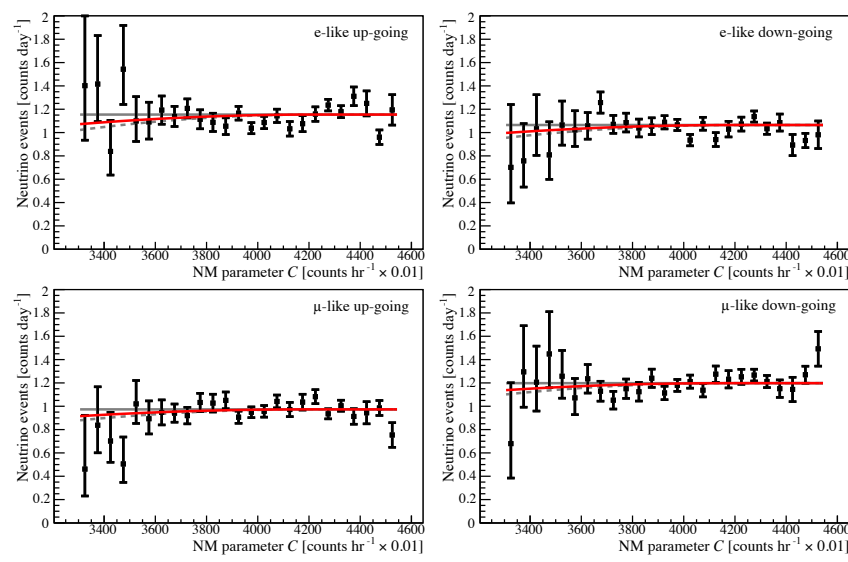

Figure 9. The test for a solar modulation correlation using the Super-K data (points). The solar correlation hypotheses are shown for no correlation ( $\alpha=0$, grey), best fit ( $\alpha=0.62$, red), and the default prediction ( $\alpha=1$, grey dotted) for each of the four data samples.

to be well correlated with the cosmic ray flux, is utilized as an indicator of the solar activity in the observed time. The expected flux changes due to solar modulation, as a function of neutrino energy and direction, are provided by the flux calculation group and introduced in this study. Figure 9 shows the observed event rate in the sub-GeV energy range, which is optimized for solar modulation search, as a function of neutron monitor count $C$. A test is performed by introducing an $\alpha$ parameter to quantify the degree of flux modulation, in which $\alpha=0$ and 1 correspond to no correlation and as predicted, respectively. The best fit gives $\alpha=0.62_{-0.58}^{+0.57}$, and the data weakly prefers the correlation with solar activity $(1.1 \sigma)$. Additional studies for the sea- sonable variation of the atmospheric neutrino due to the variation of the atmosphere density in the air are investigated. No seasonable variation is seen as expected since the change is estimated to be small $(<1 \%)$ compared to the sensitivity.

\section{Summary and Prospect}

The results of the atmospheric neutrino flux measurement have been reviewed in this paper. The $v_{\mu}$ and $v_{e}$ flux spectra, ranging from sub-GeV up to $\mathrm{PeV}$, are measured by several experiments using the variety types of the detectors. The measured fluxes are compared and tested to the flux predictions, and no significant deviations are seen. The flux component originating from $K$ decay is investigated using $v_{e}$ flux measurements above several ten $\mathrm{GeV}$, indicating larger contributions than prediction. The prompt flux, which is produced by the $D$ meson decay, has not been detected yet. Super-K data shows clear azimuthal modulation due to the geomagnetic field effect, validated in the flux simulation. The solar modulation is indicated by Super-K study but the significance is not large.

In future there are several prospects expected for the improvements of the future atmospheric neutrino flux study in the following aspects: (i) More precise flux predictions will be available using the latest cosmic ray spectrum measurement like AMS-2 and the hadronic interactions. (ii) The reduction of systematic uncertainties is necessary for further investigations of the neutrino flux, which is anticipated by future experiments using accelerator beam neutrinos. (iii) There is a possibility to measure the independent flux of neutrino and anti-neutrino, respectively, since Super-K detector is going to upgrade to SKGd by dissolving Gadolinium into the water and enhance the anti-neutrino event tagging by detecting the recoiled neutron. (iv) There are some demands for understanding of atmospheric flux below $O(100) \mathrm{MeV}$ from rare search experiments, such as dark matter, supernova relic neutrinos because the atmospheric neutrino will be a significant background in future experiments. (v) Future neutrino detectors with larger detector mass, such as HyperKamiokande, DUNE, IceCube-Gen2, PINGU, KM3Net, will improve the sensitivities of the flux measurement.

\section{References}

[1] M.G. Aartsen et al. (IceCube), Eur. Phys. J. C77, 692 (2017), 1705.07780

[2] M.G. Aartsen et al. (IceCube), Phys. Rev. D91, 122004 (2015), 1504.03753

[3] E. Richard et al. (Super-Kamiokande), Phys. Rev. D94, 052001 (2016), 1510.08127 